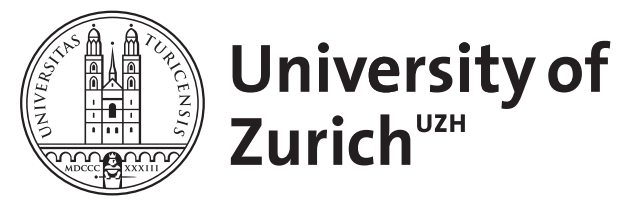

\title{
Intraossärer Zugang
}

Neuhaus, D

\begin{abstract}
Die intravenöse Pharmako- oder Infusionstherapie stellen unbestritten eine zentrale Säule in der notfallmedizinischen Versorgung, gleich ob präklinisch, in der Notfallaufnahme oder auch perioperativ, dar. Das Fehlen eines intravenösen Zugangs stellt das medizinische Personal und natürlich auch den Patienten unter Umständen vor gravierende Probleme [21]. Intravenöse Zugänge können gelegentlich patienten- oder situationsbedingt schwieriger zu etablieren sein als es unter optimalen Bedingungen möglich wäre. Solche Schwierigkeiten können in Abhängigkeit von der Dringlichkeit der Situation den Beginn der Therapie entscheidend verzögern und nicht nur den weniger erfahrenen Therapeuten erheblich unter Zeitdruck setzen. Der intraossäre Infusionszugang (IOZ) stellt in solchen Situationen die ideale Alternative zum venösen Gefäßzugang dar, insbesondere da er rasch zu erlernen, schnell zu etablieren und sicher in der Anwendung ist.
\end{abstract}

DOI: https://doi.org/10.1007/s10049-011-1445-9

Other titles: Intraosseous access

Posted at the Zurich Open Repository and Archive, University of Zurich

ZORA URL: https://doi.org/10.5167/uzh-56396

Journal Article

Published Version

Originally published at:

Neuhaus, D (2011). Intraossärer Zugang. Notfall Rettungsmedizin, 14(7):543-548.

DOI: https://doi.org/10.1007/s10049-011-1445-9 
Notfall Rettungsmed $2011 \cdot 14: 543-548$

DOI 10.1007/s10049-011-1445-9

Online publiziert: 27. Juli 2011

(c) Springer-Verlag 2011

Redaktion

T. Nicolai, München

M. Weiss, Zürich
D. Neuhaus

Anästhesieabteilung, Universitäts-Kinderkliniken, Zürich

\section{Intraossärer Zugang}

Die intravenöse Pharmako- oder Infusionstherapie stellen unbestritten eine zentrale Säule in der notfallmedizinischen Versorgung, gleich ob präklinisch, in der Notfallaufnahme oder auch perioperativ, dar. Das Fehlen eines intravenösen Zugangs stellt das medizinische Personal und natürlich auch den Patienten unter Umständen vor gravierende Probleme [21]. Intravenöse Zugänge können gelegentlich patienten- oder situationsbedingt schwieriger zu etablieren sein als es unter optimalen Bedingungen möglich wäre. Solche Schwierigkeiten können in Abhängigkeit von der Dringlichkeit der Situation den Beginn der Therapie entscheidend verzögern und nicht nur den weniger erfahrenen Therapeuten erheblich unter Zeitdruck setzen. Der intraossäre Infusionszugang (IOZ) stellt in solchen Situationen die ideale Alternative zum venösen Gefäßzugang dar, insbesondere da er rasch zu erlernen, schnell zu etablieren und sicher in der Anwendung ist.

\section{Funktionsweise des IOZ}

Die intraossäre Punktion ermöglicht die Infusion in den hämatopoetischen und daher äußerst gut durchbluteten, metaphysären Teil eines langen Röhrenknochens. Da in dieser Zone die korpuskulären Bestandteile des Bluts gebildet werden, besteht zwischen dem Markraum des Röhrenknochens und der systemischen Zirkulation ein steter Austausch. In diesem Sinne ist der Knochen quasi die "Quelle“, an welcher das Blut „entspringt“ und funktioniert daher wie eine auch bei Kälte und Hypovolämie nicht kollabierende Vene $[13,24,26]$.

Aufgrund der ,dicht gepackten“ Zellen im Markraum ist die Viskosität des eigentlichen Knochenmarks in den Marksinusoiden höher als im peripheren Blut. Daher ist bei der initialen Applikation von Substanzen in den Markraum ein leicht erhöhter Widerstand auf dem Spritzenkolben wahrzunehmen, welcher zunächst durch einen etwas erhöhten Druck bei der Injektion überwunden werden muss. Ein deutlicher Widerstandsverlust durch den Spritzenkolben zeigt dann an, dass das Knochenmark in die Systemzirkulation eingespült worden ist. Ab jetzt kann - in Abhängigkeit vom Volumen des Markraums und des Durchmessers der intraossären (IO) Kanüle - mit wenig Druck bzw. Sog sowohl Flüssigkeit injiziert als auch aspiriert werden.

Nach jeder Medikamentenapplikation muss bei der intraossären Injektion das injizierte Medikament durch einen Flüssigkeitsbolus von 5-10 ml (z. B. $\mathrm{NaCl}$ o,9\%) aus dem Markraum heraus in die Systemzirkulation eingeschwemmt werden.

Grundsätzlich können fast alle für die Behandlung von Notfallpatienten notwendigen intravenösen Medikamente in gleichen Dosierungen und Volumina auch intraossär appliziert werden [13, 24, 26]. Dies beinhaltet alle Arten von Notfallmedikamenten wie Analgetika, Anästhetika, Muskelrelaxanzien, Antiarrhythmika, Katecholamine, Infusionslösungen und Blutprodukte [3, 24]. Eine vorsorgliche Verdünnung wird lediglich im Rahmen der Anwendung hypertoner oder stark alkalischer Lösungen empfohlen, da hier möglicherweise eine erhöhte Rate an Osteomyelitiden auf dem Boden einer aseptischer Knochennekrose am Injektionsort auftreten kann $[1,10,12]$.

Hinsichtlich der Zirkulationszeit, der Bioäquivalenz und der klinischen Effektivität konnte kein Unterschied zwischen der zentralvenösen und der intraossären Applikation nachgewiesen werden [3, 25].

Die Flussgeschwindigkeit hängt bei den in der Regel großlumigen Nadeln $\left({ }_{15} \mathrm{G}\right)$ v. a. von der Resorptionsfähigkeit des jeweiligen Markraums ab, sodass ein IOZ für die alleinige „Volumeresuscitation" bei hohem Blutverlust $u$. U. bei größeren Patienten nicht ausreicht.

\section{Empfehlungen zur Indikation des $10 Z$}

Die rasche, sichere und effektive Etablierung des intraossären Infusionszugangs ist mittlerweile durch eine Vielzahl nationaler und internationaler Untersuchungen belegt $[2,5,18,20,22]$.

Die Notwendigkeit in zeitkritischen Situationen rasch eine Möglichkeit zur intravaskulären Applikation von Medikamenten und Flüssigkeiten zu schaffen, hat unterdessen zu einer klaren Stellungnahme für die Anlage eines IOZ - sowohl durch die Reanimationsgesellschaften als auch durch die nationalen Fachgesellschaften - geführt.

Der intraossäre Infusionszugang stellt somit gemäß den aktuellen Leitlinien 2010 des ERC (European Resuscitation Council) die Methode der Wahl dar, wenn bei vitaler Gefährdung von erwachsenen oder pädiatrischen Patienten (z. B. Herzkreislaufstillstand oder bedrohliche hämody- 
Tab. 1 Indikationen und klinische Beispiele für die intraossäre (IO) Kanülierung, präklinisch und perioperativ. (Mod. nach [8]).

1. Sofortindikation (frühzeitige oder primäre IO-Kanülierung)

- Atem-Kreislaufstillstand

- Akutes, fulminantes Schockgeschehen (z. B. septisch: Meningokokkensepsis)

- Hypothermie (z. B. Ertrinkungsunfall)

- Extreme Adipositas, Ödeme, großflächiges thermisches Trauma

- Kritische hämodynamische Instabilität vor oder während Anästhesieeinleitung

- Schwerer Laryngospasmus bei inhalativer Anästhesieeinleitung

- Anästhesieeinleitung bei starker Atemwegsblutung

2. Dringliche Indikation (zeitgerechte IO-Kanülierung bei erfolglosen

venösen Punktionsversuchen)

- Extreme Dehydratation bei gastrointestinalem Infekt

- Therapie starker Schmerzen (z. B.: thermisches Trauma)

- Unaufschiebbare Narkoseeinleitung beim nichtnüchternen Kind (Ileuseinleitung,

Rapid-Sequence-Induction)

- Unaufschiebbare Narkoseeinleitung beim Kind mit instabilem Kreislauf oder schwerer kardialer Insuffizienz

3. Semielektive Indikation (IO-Kanülierung bei erfolglosen venösen Punktionsversuchen und nach sorgfältiger Nutzen-Risiko-Abwägung)

- Nach inhalativer Maskeneinleitung (falls Gefäßzugang für Intervention erforderlich ist)

- Zwingende „intravenöse“ Narkoseeinleitung (z. B. bei Disposition

für eine maligne Hyperthermie)

Tab. 2 Schematische Darstellung des

Vorgehens bei der intraossären Punktion

und Kontrolle der korrekten Kanülen-

lage. (Mod. nach [3]).

1. Identifizierung der anatomischen Landmarken

2. Steriles Arbeiten und Hautdesinfektion

3. Lokalanästhesie (falls erforderlich)

4. Einbringen des intraossären Punktionssystems:

a. Punktion durch die Haut auf den Knochen ohne Rotation

b. Verifizierung eines sicheren Knochenkontakts vor Beginn der Rotation

c. Widerstandsverlust nach Penetration der Kortikalis und Eindringens in den Markraum

d. Stabiler/federnder Halt der Nadel im Knochen

e. Aspiration von Knochenmark zur Verifizierung der Kanülenlage (Cave: nicht in allen Fällen möglich)

f. Injektion von Lokalanästhetikum ( falls erforderlich)

g. Injektion eines Flüssigkeitsbolus (z. B. $5-10 \mathrm{ml} \mathrm{NaCl}$ )

5. Fixation mit dem EZ-Stabilizer ${ }^{\circledast}$ (falls EZ-IO ${ }^{\oplus}$ System verwendet)

6. Sicherung der Infusionsleitung mittels Klebestreifen an der Haut

7. Regelmäßige Kontrollen zur Erkennung einer Fehllage (Schwellung, Paravasat durch die Einstichstelle)
Tab. 3 Kontraindikationen.

(Mod. nach [8])

Als absolute Kontraindikationen gelten insbesondere Zustände, die den Erfolg einer intraossären Infusion in Frage stellen:

- Aktuelle oder kürzliche Fraktur im

Punktionsgebiet

- implantiertes Osteosynthesematerial oder kürzliche Osteosynthese-Metallentfernung im Punktionsgebiet

- Vorausgehende intraossäre Punktionsversuche $(<24-48 \mathrm{~h})$

- Gefäßverletzung proximal der Punktionsstelle

- Fehlende anatomische Landmarken

(Nur solche sind in lebensbedrohlichen Situationen zu berücksichtigen)

Als relative Kontraindikationen gelten:

- Erkrankungen des Knochens oder des Blutbildungssystems

- Intrakardialer Rechts-Links-Shunt (Gefahr einer paradoxen Knochenmark- oder Fettembolie)

(Diese relativen Kontraindikationen sind nur von Bedeutung für Situationen, in denen keine lebensbedrohliche Situation vorliegt) namische Instabilität) die Anlage eines intravenösen Zugangs misslingt oder Schwierigkeiten bei der Anlage die zeitgerechte Versorgung des Patienten verzögern („Sofortindikation“). Als Richtlinie wird hier oftmals vorgeschlagen, spätestens nach drei misslungenen periphervenösen Punktionsversuchen oder nach 90-120 s auf einen intraossären Infusionszugang zu wechseln $[4,9,11,26]$. Außerdem kann bei Kindern mit offensichtlich schwierigsten Venenverhältnissen auch primär eine intraossäre Infusion angelegt werden, wenn dies zur Sicherung der Vitalfunktionen, aber ebenso auch zur Analgesie bei stärksten Schmerzen angebracht erscheint (z. B. Situationen wie ausgeprägte Adipositas, generalisierte Ödeme, großflächige thermische Verletzungen, Hypovolämie oder Hypothermie). In Reanimationssituationen wird vielerorts der IOZ an der Tibia bei Kindern und Erwachsenen als Primärzugang gewählt, um die Herz-Lungen-Wiederbelebungsmaßnahmen an der oberen Körperhälfte weniger zu behindern.

Aber auch im klinischen Alltag kann eine schwierige Venensituation das behandelnde Team vor große Schwierigkeiten stellen. In den neuesten Handlungsempfehlungen des Wissenschaftlichen Arbeitskreises Kinderanästhesie (WAKKA) der DGAI (Deutsche Gesellschaft für Anästhesie und Intensivmedizin) sind die Indikation für den intraossären Infusionszugang der Leitlinien des ERC in erweiterter Form übernommen und auf die perioperative Versorgung von Kindern in Form von Sofort-, dringlichen und semielektiven Indikationen“ übertragen ([8, 20], ( Tab. 1).

Die Entscheidung zur Anlage eines IOZ ist jedoch wie jede medizinische Maßnahme auch, abgesehen von vital bedrohlichen Situationen, immer einer individuellen Nutzen-Risiko-Abwägung zu unterziehen und sollte daher nicht leichtfertig getroffen werden.

\section{Technik und Material}

Die Punktionsstelle der ersten Wahl für einen IOZ beim Kind ist die proximale Tibia ([14, 27], • Abb. 1). Hier wird von der Tuberositas tibiae, je nach Körperlänge des Kindes, 1-2 cm nach distal palpiert 
und von dort ca. $1 \mathrm{~cm}$ nach medial. Dort findet sich die flache, anteromediale Tibiavorderfläche, die sich für die Punktion mit automatischen Systemen für alle Altersgruppen hervorragend eignet. Bei sehr kleinen Kindern und Säuglingen kann die Tuberositas tibiae noch nicht palpabel sein. In diesen Fällen dient die Unterkante der Patella als Ausgangspunkt für die Orientierung.

Die Punktionsstelle der zweiten Wahl ist die distale Tibia. Ausgangspunkt für die Orientierung ist hier der höchste Punkt des medialen Malleolus. Die Punktionstelle liegt von dort 1-3 cm kranial. An dieser Punktionsstelle ist die Kortikalis dünner und daher mit manuellen Systemen (z. B. COOK ${ }^{\circledast}$, Cook Critical Care, Bloomington, IN, USA) auch noch bei größeren Kindern und Erwachsenen leichter zu penetrieren [17]. Bei automatischen Systemen (z. B. EZ-IO ${ }^{\circ}$, Vidacare, San Antonio, Texas, USA) spielt diese Überlegung keine Rolle. Beide Punktionsstellen können alternierend am selben Bein verwendet werden, sollte an der anderen Stelle eine Kontraindikation bestehen (z. B. Fraktur, vorangegangene IOZ-Punktion $<24 \mathrm{~h}$, Osteosynthesematerial).

In einer Untersuchung zur Häufigkeit des Einsatzes des IOZ im pädiatrischen Rettungsdienst musste lediglich bei einem von 77 Kindern $(1,3 \%)$ auf die obere Extremität ausgewichen werden, da eine Anlage an der unteren Extremität aufgrund einer beidseitigen Amputationsverletzung nicht möglich war [22].

Weitere alternative Punktionsstellen sind der anteromediale proximale $\mathrm{Hu}$ merus und das distale Femur oberhalb der Patella. Das Sternum als intraossärer Punktionsort ist bei Kindern wegen der Verletzungsgefahr mediastinaler Strukturen obsolet.

\section{( Die eingesetzte Technik hängt von dem im eigenen Haus zur Verfügung stehenden Material ab}

Welche Technik zum Einsatz kommt, hängt letztlich von dem im eigenen Haus zur Verfügung stehenden Material ab, mit welchem der Anwender zwingend vertraut sein muss. Momentan ist der Trend $\mathrm{zu}$ beobachten, weg von den manuellen

Notfall Rettungsmed $2011 \cdot 14: 543-548$ DOI 10.1007/s10049-011-1445-9

(c) Springer-Verlag 2011

\section{Neuhaus \\ Intraossärer Zugang}

\section{Zusammenfassung}

Sowohl im präklinischen wie auch im klinischen Alltag kann eine schwierige Venensituation den Beginn einer dringend notwendigen intravenösen Therapie empfindlich verzögern. Der intraossäre Infusionszugang (IOZ) als Gefäßzugang stellt eine rasche und zuverlässige Alternative zum konventionellen intravenösen Zugang bei Patienten mit schwierigem oder unmöglichem Venenzugang und dringend benötigter intravaskulärer Therapie dar. Nach der intraossären Applikation stehen alle für die Notfallsituation gewünschten Medikamente und Flüssigkeiten schnell, sicher und effektiv am Wirkort zur Verfügung. Bei korrekter und kurzzeitiger Anwendung überwiegt der Nutzen eines IOZ die möglichen Komplikationen bei Weitem. Der Einsatz des IOZ erstreckt sich da-

\section{Intraosseous access}

\section{Abstract}

Difficult venous access may significantly delay the onset of an urgent intravenous therapy, not only in prehospital emergency patients but also in routine clinical practice. Intraosseous infusion has become established as a fast and reliable alternative route for peripheral intravenous puncture in patients with difficult or failed venous access needing immediate intravenous therapy. Intraosseous administration of almost all emergency drugs and fluids results in a fast, safe and reliable effect at the receptor organ. Benefits of appropriate indications and time-limited application of the intraosseous technique far outweigh the possible complications. The indications for the intraosseous infusion technique have now been extended from an im- her mittlerweile von der "Sofortindikation“ beim schwerst kranken oder schwerst verletzten Notfallpatienten über die „dringliche" Indikation bei dem im Augenblick noch stabilen Patienten mit z. B. unaufschiebbarer Narkoseeinleitung bis hin zur ,semielektiven" Indikation beim elektiven Patienten nach sorgfältiger Nutzen-Risiko-Abwägung. Für den sicheren und effektiven Einsatz des intraossären Infusionszugangs müssen die Indikationen festgelegt, das medizinische Personal geschult und instruiert sein sowie adäquates Material jederzeit griffbereit in Patientennähe zur Verfügung stehen.

\section{Schlüsselwörter}

Intraossär · Infusion · Anästhesie · Kinder · Schwieriger Gefäßzugang mediate indication in critically ill or injured patients to an urgent indication in patients still stable but with a not-to-be-delayed surgery and even to a semi-elective indication in certain routine patients after crucial risk-benefit analysis. For a safe and effective application of the intraosseous infusion technique, the indications must be defined, medical personnel have to be thoroughly trained and instructed and the material for intraosseous puncture has to be made readily available at the patient bedside.

\section{Keywords} Intraosseous · Infusion · Anaesthesia · Children $\cdot$ Difficult venous access 

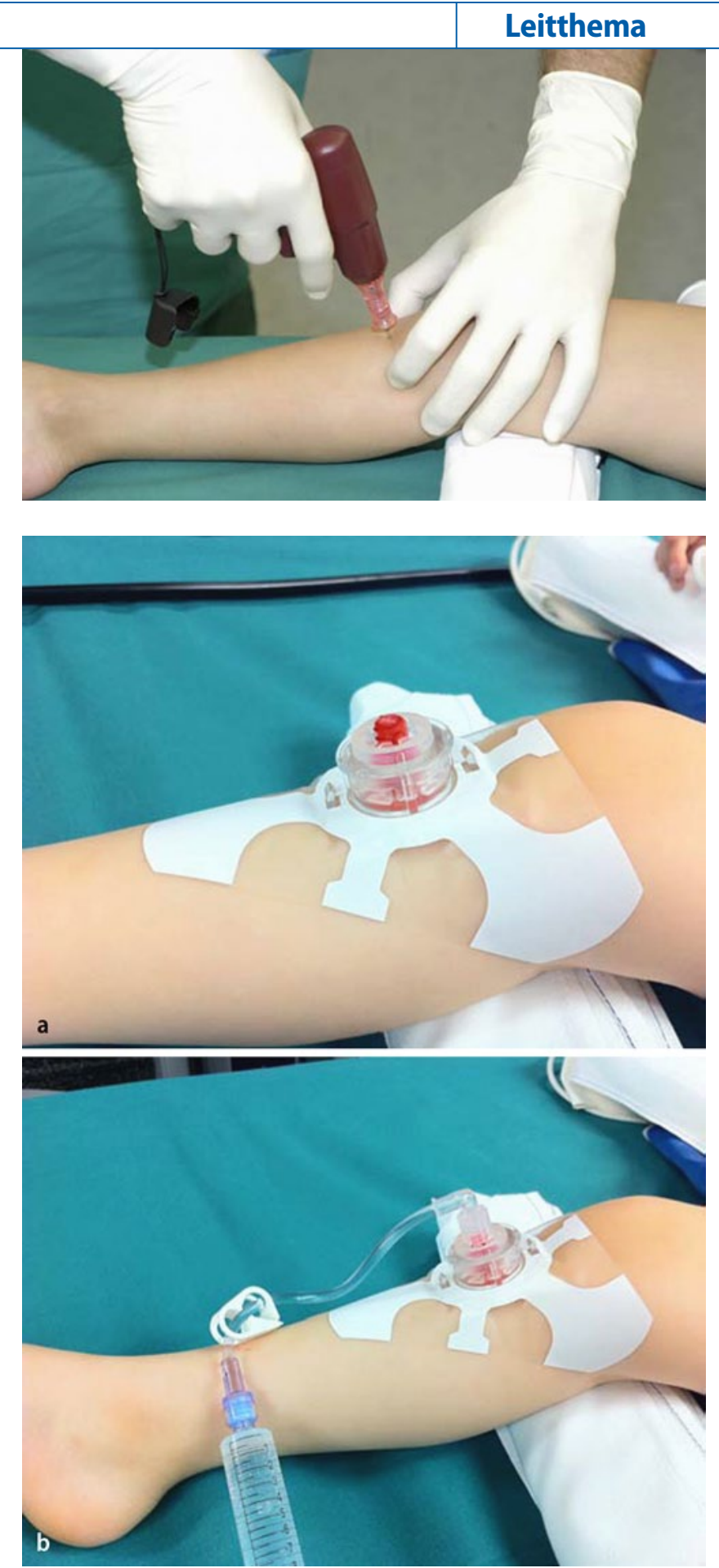

Abb. $1<$ Intraossärer Infusionszugang mit dem EZ-IO ${ }^{\otimes}$ System

Abb. $2<$ EZ-Stabilizer $^{\oplus}$. a vor Konnektion, b nach Konnektion an passende Infusionsverlängerung (Infusionsleitung noch mit Pflasterzug befestigen!)
Systemen, bei welchen die Nadeln unter Drehbewegungen händisch in den Knochen eingebracht werden müssen, hin zu automatischen "akkubohrerähnlichen“ Systemen, die es ermöglichen, rasch einen exakt zylindrischen Bohrkanal zu schaffen.

Unabhängig vom verwendeten System ist in jedem Fall ein streng aseptisches Vorgehen unabdingbar. Hier müssen 3 Punkte beachtet werden:
- Vor dem Einbringen der Kanüle muss eine sterile Wischdesinfektion mit ausreichend langer Einwirkzeit erfolgen. Gerade bei vitaler Indikation kann auf Lochtuch und Mundschutz verzichtet werden, jedoch darf die Punktionsstelle nicht nach erfolgter Hautdesinfektion nochmals mit einem „unsterilen“ Finger palpiert werden („No-touch-Technik“). Sterile Handschuhe müssen lediglich bei manuellen Systemen getragen werden, da diese für ein sicheres Einbringen per se an der Nadel selber angefasst werden müssen.

- Mindestens ebenso wichtig ist nach erfolgreicher Anlage des IOZ der korrekte und hygienische Umgang mit der Infusionslösung und allen Konnektions- und Injektionsadaptern (z. B. Dreiwegehahn), analog zum Umgang mit zentralvenösen Langzeitverweilkathetern (z. B. HickmanKatheter). Grundsätzlich sollte der IOZ daher nur solange in situ verbleiben wie er unabdingbar ist, höchstens jedoch 12-24 h [14, 20, 24].

- Die Einmalgabe eines geeigneten Antibiotikums im Sinne einer Single-shot-Prophylaxe (z. B. Cefuroxim, Cephazolin) direkt durch die IO-Nadel ist analog der AB-Prophylaxe bei Osteosynthesen zu erwägen, gemäß der WAKKA-Handlungsempfehlung insbesondere jedoch bei zeitkritischen Notfallsituationen (Unterschreiten der Einwirkzeit des Desinfektionsmittels) und fraglich ausreichender lokaler Hygiene indiziert ([8]; Ablaufschema der intraossären Punktion s. - Tab. 2).

Die unbemerkte Dislokation der Nadel durch Fehlpunktion, Fraktur oder Zug an der Infusionsleitung kann zum Verlust der Infusion oder auch zum Paravasat führen. Anders als bei dem Paravasat einer periphervenösen Nadel (z. B. am Handrücken) kann bei einer intraossären Infusion das Paravasat, z. B. bei Perforation der Gegenkortikalis, unbemerkt in eine tiefe Muskelloge laufen. Daher müssen nebst guter Fixation - der Punktionsort und die Extremität während der laufenden Infusion, insbesondere bei bewusstlosen oder analgosedierten Patienten, regelmäßig auf Zeichen einer Dislokation und Schwellung hin untersucht werden. Schwerwiegende Schädigungen sind aufgrund dieser Komplikation berichtet worden [23].

EZ-IO ${ }^{\oplus}$ bietet bezüglich der Stabilität der eingebrachten IO-Nadel mit dem sog. „EZ-Stabilizer “ eine vielversprechende Verbesserung der Nadelfixation (• Abb. 2). 
Eine lokale Betäubung der Injektionsstelle beim bewusstseinsklaren Patienten muss individuell aus der jeweiligen Situation heraus entschieden werden. Untersuchungen zur Schmerzhaftigkeit einer intraossären Punktion berichten einen Schmerzindex von 2,3 $( \pm 2,8)$ auf der „Visuellen Analogskala“ (VAS) für die eigentliche Punktion mit automatischen Systemen. Da auch das Endost sensibel versorgt ist, werden außerdem sowohl Injektion als auch Aspiration als schmerzhaft empfunden, häufig sogar schmerzhafter als die eigentliche Punktion (VAS: 3,2 $\pm 3,5 ;$ [16]).

In jedem Fall muss erwogen werden, dass eine gut gemeinte lokale Betäubung des Periosts dem Kind so unangenehm sein kann, dass ein etwaiger Vertrauensbonus für die eigentliche intraossäre Punktion verspielt ist.

\section{Aufklärung}

Nach bereits erfolgter IOZ-Anlage muss im Nachhinein mit dem Patienten oder den Angehörigen/Eltern ein informelles Gespräch über die durchgeführte Maßnahme und ihre Notwendigkeit erfolgen.

Bei elektiven Patienten mit bekannten oder erwartet schwierigen Venenverhältnissen im Rahmen einer Anästhesie empfiehlt sich die Erwähnung der Möglichkeit eines IOZ und seiner typischen, wenn auch seltenen Komplikationen bereits beim Prämedikationsgespräch.

\section{Komplikationen}

Wie viele invasive medizinische Maßsnahmen hat auch der intraossäre Infusionszugang typische Komplikationen, welche jedoch selten zu klinisch relevanten Schwierigkeiten führen.

\section{(? Die häufigste Komplikation ist die Dislokation und die relevanteste die Osteomyelitis}

Die häufigste Komplikation ist die Dislokation und die relevanteste stellt die Osteomyelitis dar. Obwohl davon ausgegangen werden kann, dass bei steriler Arbeitstechnik, aseptischem Umgang mit dem Infusionssystem und kurzer Verweildauer der Kanüle die Osteomyelitis eine aus- gesprochene Rarität ist [15], kann eine gewisse Dunkelziffer nicht ausgeschlossen werden. Am ehesten wird ihr Auftreten bei Immunsuppression, septischen Schockzuständen mit Bakteriämie oder der Anwendung unverdünnter, hyperonkotischer Lösungen erwartet $[3,8]$.

Bei korrekter Identifikation der Punktionsstelle ist eine Verletzung der Epiphysenfuge bei Kindern im Wachstumsalter nahezu ausgeschlossen. Entsprechend finden sich darüber keine Hinweise in der aktuellen Literatur [6]. Ebenso selten scheinen Mikroembolisationen eine klinische Relevanz zu entwickeln. Gegebenenfalls muss eine erhöhte Rechtsherzbelastung bei vorbestehendem pulmonalem Hypertonus berücksichtigt werden sowie eine paradoxe Embolie bei kardialem Rechts-Links-Shunt.

Unsachgemäße Handhabung kann bei Verwendung manueller Systeme zum Verbiegen oder Brechen der Punktionskanüle führen, in wenigen Fällen sogar zu Knochenbrüchen [19]. Automatische Systeme scheinen diesbezüglich Vorteile zu bieten.

Der Verlust der korrekt liegenden Kanüle (z. B. bei Umlagerung), Fehllage, Perforation der Gegenkortikalis mit dem potenziellen Risiko der Entwicklung eines Kompartmentsyndroms an der betroffenen Extremität [23] sind die praktischen Probleme, welchen nur mit Übung und Achtsamkeit entgegengewirkt werden kann.

\section{Kontraindikationen}

In vital bedrohlichen Situation gibt es keine Kontraindikation - außer, sie verhindern eine erfolgreiche Infusion! Bei dringlichen oder semielektiven Indikationen müssen allfällige „nichtabsolute“ Kontraindikationen beachtet werden (• Tab. 3).

\section{Vorbereitung und praktische Übung}

Regelmäßiges Training am Phantom, industriellen Übungsknochen oder auch Tierknochen ist wichtig, um einerseits "Hemmungen“ abzubauen und andererseits das richtige Gefühl für das Bohren in einen Knochen zu entwickeln. Einerseits ist zwar die Ersterfolgsrate auch beim wenig geübten Anwender hoch [16], an- dererseits wird aber diese Maßnahme zu selten, dann jedoch in der Regel in einer Stresssituation durchgeführt. Daher sollte der Anwender mit dem in seiner Institution vorgehaltenen Material vertraut sein, die notwendige sterile Arbeitsweise auch unter Druck korrekt einhalten und ein gewisses „Gefühl“ für das Bohren in den Knochen entwickeln. Da die meisten Notfallhelfer aus den Bereichen der Anästhesie und Akut- oder Intensivmedizin und nicht beispielsweise aus der Orthopädie stammen, sind sie mit der Materie, eine Knochenkompakta (Substantia corticalis) zu durchbohren aus eigener Erfahrung nicht vertraut. Hier besteht, besonders beim Anfänger, häufig eine große psychische und technische Hemmschwelle, welche sich idealerweise nur durch Aufklärung und regelmäßige Übung an Knochenmodellen aufheben lässt [7].

Mancherorts wird bei Säuglingen mit onkologischen Erkrankungen die diagnostische Knochenmarkpunktion bevorzugt an der Tibia durchgeführt. Da diese Kinder hierfür ohnehin eine Allgemeinanästhesie benötigen, kann sich vielleicht in Rücksprache mit den zuständigen Onkologen auch eine weitere Möglichkeit für den Anästhesisten ergeben, die Durchführung des intraossären Infusionszugangs unter optimalen Bedingungen zu erlernen.

\section{Fazit für die Praxis}

- Der intraossäre Infusionszugang hat mittlerweile nicht nur einen festen Stellenwert in der Akutversorgung schwer kranker und verletzter Patienten, sondern auch - bei reflektierter Indikationsstellung - im perioperativen Bereich einiger elektiver Patienten.

- Bei korrekter und zeitgerechter Indikationsstellung sowie technisch und hygienisch einwandfreier Durchführung überwiegt der Nutzen etwaige Risiken bei weitem.

- Voraussetzung hierfür sind klare Indikationsvorgaben, die unmittelbare Verfügbarkeit des Materials am Ort der Patientenversorgung und regelmäßige Schulung und Training des anwendenden Personals. 


\section{Korrespondenzadresse}

\section{Dr. D. Neuhaus}

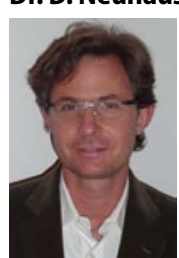

Anästhesieabteilung,

Universitäts-Kinderkliniken

Steinwiesstraße 75, 8032 Zürich

Schweiz

diego.neuhaus@kispi.uzh.ch

Interessenkonflikt. Der korrespondierende Autor gibt an, dass kein Interessenkonflikt besteht.

\section{Literatur}

1. Alam H, Punzalan C, Koustova E et al (2002) Hypertonic saline: intraosseous infusion causes myonecrosis in dehydrated swine model of uncontrolled hemorrhagic shock. J Trauma 52:18-25

2. Banerjee S, Singhi S, Singh S, Singh M (1994) The intraosseous route is a suitable alternative to intravenous route for fluid resuscitation in severely dehydrated children. Indian Pediatr 31:1511-1520

3. Bernhard M, Gräsner J-T, Gries A et al (2010) Die intraossäre Infusion in der Notfallmedizin. Empfehlungen des Wissenschaftlichen Arbeitskreises Notfallmedizin und des Wissenschaftlichen Arbeitskreises Kinderanästhesie der DGAI. Anästh Intesivmed 51:615-620

4. Biarent D, Bingham R, Eich C et al (2010) European Resuscitation Council Guidelines for Resuscitation 2010. Section 6: Paediatric Life Support. Resuscitation 81:1364-1388

5. Brenner T, Gries A, Helm M, Bernhard M (2009) Intraosseous infusion systems in the prehospital setting. Resuscitation 80:607

6. Claudet I, Baunin C, Laporte-Turpin E et al (2003) Long-term effects on tibial growth after intraosseous infusion: a prospective, radiographic analysis. Pediatr Emerg Care 19:397-401

7. Eich C, Russo S, Timmermann A et al (2006) Neue Perspektiven in der simulatorunterstützten Ausbildung in Kinderanästhesie und Kindernotfallmedizin. Anaesthesist 55:179-184

8. Eich C, Weiss M, Neuhaus D et al (2011) Handlungsempfehlungen zur intraossären Infusion in der Kinderanästhesie. Anästh Intesivmed 52:46-52

9. Eich C, Weiss M, Neuhaus D et al (2010) Die intraossäre Infusion in der Kindernotfallmedizin und Kinderanästhesie. Anästh Intesivmed 51:75-81

10. Fiser DH (1990) Intraosseous infusion. N Engl J Med 322:1579-1581

11. Fowler $R$, Gallagher JM, Isaacs $S$ et al (2007) The role of intraosseous vascular access in the out-ofhospital environment (resource document to NAEMSP Position Statement). Prehosp Emerg Care 11:63-66

12. Guy J, Haley K, Zuspan S (1993) Use of intraosseous infusion in the pediatric trauma patient. J Pediatr Surg 23

13. Helm M, Fischer S, Hauke J et al (2008) Invasive Techniken in der Notfallmedizin. Notfall Rettungsmed 11:317-324

14. Helm M, Gries A, Fischer S et al (2005) Invasive techniques in emergency medicine. III. Intraosseous punction-an alternative vascular access in paediatric emergencies. Anaesthesist 54:49-56

15. Henson NL, Payan JM, Terk MR (2011) Tibial subacute osteomyelitis with intraosseous abscess: an unusual complication of intraosseous infusion. Skeletal Radiol 40:239-242
16. Horton MA, Beamer C (2008) Powered intraosseous insertion provides safe and effective vascular access for pediatric emergency patients. Pediatr Emerg Care 24:347-350

17. Iserson K (1989) Intraosseous infusion in adults. J Emerg Med 7:587-591

18. Leidel BA, Kirchhoff C, Bogner V et al (2009) Is the intraosseous route fast and efficacious compared to conventional central venous catheterization in adult patients under resuscitation in the emergency department? A prospective observational pilot study. Patient Saf Surg 3:24

19. Neuhaus D, Henze G, Frotzler A, Weiss M (2010) Stellenwert der intraossären Infusion in der Kinderanästhesie. Eine internetbasierte Umfrage. Anästh Intesivmed 51:328-334

20. Neuhaus D, Weiss M, Engelhardt T et al (2010) Semi-elective intraosseous infusion after failed intravenous access in pediatric anesthesia. Paediatr Anaesth 20:168-171

21. Orlowski JP (1984) My kingdom for an intravenous line. Am J Dis Child 138:803

22. Sommer A, Weiss M, Deanovic D et al (2011) Einsatz der intraossären Infusion im pädiatrischen Notarztdienst. Analyse von Notarzteinsätzen 1990-2009. Anaesthesist 60:125-131

23. Taylor CC, Clarke NM (2011) Amputation and intraosseous access in infants. BMJ 342:d2778

24. Tobias JD, Ross AK (2010) Intraosseous infusions: a review for the anesthesiologist with a focus on pediatric use. Anesth Analg 110:391-401

25. Von Hoff DD, Kuhn JG, Burris HA $3^{\text {rd }}$, Miller $L$ (2008) Does intraosseous equal intravenous? A pharmacokinetic study. Am J Emerg Med 26:31-38

26. Weiss M, Gächter-Angehrn J, Neuhaus D (2007) Intraossäre Infusionstechnik. Notfall Rettungsmed 10:99-116

27. Weiss M, Henze G, Eich C, Neuhaus D (2009) Intraoseous infusion - an important technique in paediatric anaesthesia [article in German]. Anaesthesist 58:863-875

\section{Forscher bestimmen Zeitpunkt eines Schlaganfalls}

Die Information über den Zeitpunkt des Symptombeginns spielt eine entscheidende Rolle in der Akutbehandlung des ischämischen Schlaganfalls.

Eine Forschergruppe des Universitätsklinikums Hamburg-Eppendorf (UKE) hat nun mittels Magnetresonanztomographie (MRT) eine neue Methode entwickelt, mit der sich der Zeitpunkt des Schlaganfalls eingrenzen lässt. Die Forscher untersuchten 643 Patienten aus 10 Schlaganfallzentren. Sie konnten zeigen, dass die Kombination zweier MRTTechniken (diffusion-weighted imaging (DWI) und fluid-attenuated inversion recovery (FLAIR) MRT) Rückschlüsse auf das Alter eines Hirninfarkts zulässt. Das Vorliegen eines bestimmten Musters erlaubte die Identifikation von Patienten, deren Schlaganfall weniger als 4,5 Stunden zurück lag und die damit im Zeitfenster für eine effektive und sichere Thrombolyse lagen. Die Treffsicherheit für die Bestimmung dieser Patientengruppe lag bei $87 \%$.

Die Untersuchung ist Grundlage für die europäische Studie "WAKE-UP", die von der EU mit 11,6 Millionen Euro gefördert und von den Wissenschaftlern des UKE geleitet wird.

Literatur: Thomalla G, Cheng B, Ebinger M et al (2011) DWI-FLAIR mismatch for the identification of patients with acute ischaemic stroke within $4.5 \mathrm{~h}$ of symptom onset (PRE-FLAIR): a multicentre observational study.

The Lancet Neurology, doi: 10.1016/S1474-4422(11)70192-2

Quelle: Universitätsklinikum Hamburg-Eppendorf, www.uke.de 